TRAVAIL

ET EMPLOI

\section{Travail et Emploi}

135 | juillet-septembre 2013

Expérimentations de terrain et politiques publiques du travail et de l'emploi. Apports récents et mises en perspective

\title{
Évaluation par expérimentation aléatoire de dispositifs de reclassement innovants pour les demandeurs d'emploi
}

Randomized experiment and evaluation of innovative placing schemes for the unemployed

Marc Ferracci et Florine Martin

\section{(2) OpenEdition}

Journals

Édition électronique

URL : http://journals.openedition.org/travailemploi/6051

DOI : 10.4000/travailemploi.6051

ISSN : 1775-416X

Éditeur

DARES - Ministère du Travail

Édition imprimée

Date de publication : 15 septembre 2013

Pagination : 15-26

ISSN : 0224-4365

Référence électronique

Marc Ferracci et Florine Martin, «Évaluation par expérimentation aléatoire de dispositifs de reclassement innovants pour les demandeurs d'emploi », Travail et Emploi [En ligne], 135 | juilletseptembre 2013, mis en ligne le 01 juillet 2015, consulté le 01 mai 2019. URL : http:// journals.openedition.org/travailemploi/6051; DOI : 10.4000/travailemploi.6051 


\title{
Évaluation par expérimentation aléatoire de dispositifs de reclassement innovants pour les demandeurs d'emploi
}

\author{
Marc Ferracci(*), Florine Martin ${ }^{(* *)}$
}

L'amélioration des dispositifs d'accompagnement et de placement des chômeurs est un enjeu essentiel des politiques actives du marché du travail. Alors que de nombreux travaux académiques s'intéressent aux effets d'interventions centrées sur les demandeurs d'emploi, cet article se propose d'évaluer des méthodes de travail innovantes visant à accroître l'efficacité des conseillers chargés du placement. Au moyen d'une expérimentation aléatoire sont ainsi testés deux dispositifs distincts. Le premier a pour objectif d'inciter les conseillers à mieux organiser leur temps de travail en leur permettant de concentrer ponctuellement leurs efforts sur un nombre réduit de demandeurs d'emploi. Le second consiste à augmenter les ressources dédiées à la recherche d'offres d'emploi et au positionnement des candidats sur ces offres. Les résultats obtenus montrent que les deux dispositifs peuvent améliorer le taux de sortie du chômage, mais que cet effet positif ne vaut pas pour toutes les populations de demandeurs d'emploi, ce qui soulève la question des effets externes de ce type d'actions.

Les budgets des politiques d'emploi ne sont pas extensibles. En 2012, les crédits alloués dans la loi de finances à la mission «Travail et Emploi» étaient en baisse de $11 \%$ par rapport à 2011, pour un total de 10,2 milliards d'euros. Quant aux subventions allouées à Pôle Emploi, elles étaient maintenues au même niveau qu'en 2010 et 2011 alors même que le nombre de demandeurs d'emploi à accompagner est toujours plus important ${ }^{(1)}$. Ces restrictions budgétaires accroissent, s'il en était besoin, la nécessité d'une évaluation systématique des actions d'accompagnement des chômeurs. Les contraintes actuelles imposent d'imaginer des méthodes permettant d'accroître l'efficacité du reclassement à moyens constants.

Dans cette perspective, cet article présente les résultats d'une expérimentation sociale visant à améliorer l'efficacité de l'accompagnement des demandeurs d'emploi en mettant l'accent sur les méthodes employées par les conseillers plutôt que sur un surcroît de moyens dédiés à l'accompagnement en tant que tel (la fréquence des entretiens par exemple). À l'aide de données originales

(*) LEMNA-Université de Nantes, Crest-ENSAE, LIEPPSciences Po, Chaire de sécurisation des parcours professionnels ; marc.ferracci@ensae.fr

(**) Érudite, Université Paris-Est Marne-la-Vallée, chargée d'études chez Sodie; florine.martin@sodie.com

(1) Fin juin 2012, les statistiques de Pôle Emploi présentées par la Direction de l'animation de la recherche, des études et des statistiques (Dares) montrent une augmentation de 6,9\% du nombre de demandeurs d'emploi inscrits en catégories A, B et $\mathrm{C}$ sur une année glissante (DARES, 2012). collectées par un opérateur privé de placement (OPP) œuvrant pour le compte de Pôle Emploi, l'entreprise Sodie, deux dispositifs innovants sont ainsi testés. Au regard d'autres évaluations existant en France et à l'étranger, la particularité de ces dispositifs consiste à cibler les conseillers chargés du placement, et non les demandeurs d'emploi euxmêmes. Le premier vise à inciter les conseillers à mieux organiser leur temps de travail, en concentrant ponctuellement leurs efforts sur un nombre réduit de demandeurs d'emploi. Le second consiste à accroître les ressources dédiées à la recherche d'offres d'emploi et au positionnement des candidats sur ces offres. Les résultats obtenus montrent que les deux dispositifs peuvent augmenter le taux de sortie du chômage, mais que cet effet positif ne vaut pas pour toutes les populations de demandeurs d'emploi. Ceci soulève la question des effets externes de ce type d'actions.

\section{Éléments de littérature}

Il existe de nombreuses évaluations quantitatives de l'effet d'un accompagnement renforcé des demandeurs d'emploi, comparé à celui d'un suivi classique. En France, depuis le début des années 2000 et plus encore depuis la réforme constitutionnelle du 28 mars 2003 autorisant les expérimentations aléatoires au sein des collectivités territoriales, ce type d'évaluation a pris un certain essor. Dans leur méta-analyse relative aux politiques actives d'emploi, David CARD, Jochen 
Kluve et Andrea Weber (2010) montrent ainsi que les programmes d'accompagnement ont généralement une efficacité supérieure aux contrats aidés dans le secteur public, et exercent leurs effets plus rapidement que les programmes de formation professionnelle. Sur données françaises, plusieurs études mettent en évidence l'impact positif d'un accompagnement renforcé sur les perspectives de retour à l'emploi des chômeurs qui en bénéficient. Exploitant les données du fichier historique de l'Agence nationale pour l'emploi (ANPE), Bruno Crépon, Muriel Dejemeppe et Marc Gurgand (2005) montrent les effets hétérogènes de différents programmes d'accompagnement alternatifs sur le retour à l'emploi, mais aussi sur la durée de l'emploi retrouvé. Au niveau international, les évaluations de ce type sont nombreuses, et leurs résultats globalement convergents. Récemment Pathric HäGglund (2009) souligne, à partir d'une expérimentation sur données suédoises, l'effet positif de l'accompagnement renforcé sur la durée passée au chômage. Richard Blundell et ses coauteurs (2004), de leur côté, évaluent un dispositif d'accompagnement dédié aux jeunes chômeurs britanniques, le programme «New Deal for the young unemployed» et trouvent également des résultats positifs. Ainsi les études sur données françaises tendent à confirmer les résultats issus de la littérature étrangère (2), qui montrent que l'accompagnement renforcé permet bien d'accroître le taux de retour à l'emploi des bénéficiaires et ce, quel que soit le type de public ou d'opérateur.

Néanmoins, les dépenses consécutives à la mise en place de tels dispositifs sont importantes et doivent être considérées au regard des bénéfices engendrés ${ }^{(3)}$. Certaines études récentes ont cherché à évaluer le rapport coût/bénéfice des dispositifs d'accompagnement. C'est le cas du travail de CRÉPON et ses coauteurs (2011), qui ont mené ce type d'analyse sur un dispositif d'accompagnement renforcé des bénéficiaires du revenu minimum d'insertion (RMI) par un opérateur privé de placement. Si leur travail met bien en évidence l'impact positif du dispositif sur les perspectives de retour à l'emploi des bénéficiaires et les transferts sociaux, il montre également que ce programme n'est pas toujours rentable, dans la mesure où les gains engendrés ne parviennent pas à compenser ses coûts. Dans un autre contexte, l'article de Hainmueller et al. (2011) sur données allemandes aboutit à des résultats différents. Ces

(2) Se reporter à FERRACCI (2007) pour une revue de littérature sur l'efficacité des réformes du service public de l'emploi.

(3) Ceci est d'autant plus important que les travaux à la fois théoriques et empiriques relatifs à l'externalisation des services publics montrent que les prestataires privés adoptent des stratégies qui leur permettent de maximiser leur profit, compte tenu des incitations financières qui leur sont données. Dans le prolongement de leur travail d'évaluation, BeHaghel et al. (2012) s'intéressent aux déterminants de l'efficacité des prestataires, et concluent que les incitations financières jouent un rôle essentiel. derniers trouvent un effet positif de la baisse de la taille des portefeuilles des conseillers sur la durée passée au chômage, qui s'explique à la fois par une augmentation des sanctions et des offres d'emploi proposées aux demandeurs d'emploi accompagnés. Contrairement à l'étude de CRÉPON et al. (2011), ils concluent qu'à long terme les coûts liés à l'embauche de conseillers supplémentaires sont compensés par les effets positifs de la baisse de la taille des portefeuilles.

Si elle améliore généralement le reclassement, il n'apparaît donc pas évident que l'augmentation des moyens dédiés à l'accompagnement ait systématiquement un rapport coût/bénéfice positif. Ceci invite à envisager, en marge ou au sein du service public de l'emploi (SPE), la mise en œuvre de méthodes d'accompagnement alternatives, et potentiellement plus efficientes. Cette contrainte d'efficience a contribué récemment à un recours croissant aux prestataires externes chargés de concourir au placement, en appui du SPE, les OPP étant parfois présentés comme plus réactifs et plus innovants dans leurs méthodes d'accompagnement. Alors que l'externalisation des services de placement est désormais largement répandue dans les pays de l'OCDE (Organisation de coopération et de développement économiques), la France s'est engagée dans cette voie assez tardivement, au début des années 2000.

Certaines évaluations ont depuis cherché à mesurer la plus-value apportée par les OPP, en la comparant à celle de dispositifs spécifiques du service public de l'emploi. L'étude de CRÉPON et al. (2011) déjà citée montre ainsi, grâce à une évaluation randomisée à double niveau, qu'un accompagnement renforcé des jeunes diplômés demandeurs d'emploi au chômage, réalisé par un prestataire externe (association ou opérateur privé de placement), permet d'accélérer leur accès à un emploi durable. À l'aide d'une expérimentation contrôlée, Luc BeHAGHEL et ses coauteurs (2012) obtiennent des résultats similaires : un accompagnement renforcé des demandeurs d'emploi, via un OPP ou une cellule spécifique mise en place par l'ANPE («Cap vers l'entreprise»), améliore les perspectives d'emploi des bénéficiaires, indemnisés ou non. Plus précisément, l'étude de Behaghel et al. (2012) met en évidence un impact plus grand et plus précoce de la cellule «Cap vers l'entreprise» par rapport aux opérateurs privés de placement.

L'expérimentation contrôlée réalisée par Behaghel et al. (2012) a été couplée à une monographie de terrain menée auprès des acteurs de l'accompagnement (OPP et CVE), et à une enquête téléphonique auprès d'un échantillon représentatif de demandeurs d'emploi ayant bénéficié d'un accompagnement renforcé ou d'un accompagnement classique (Gratadour, Le Barbanchon, 
2009; Capelier, Mizrahi, 2008). L'étude, de nature qualitative, a permis de révéler les proximités de l'accompagnement renforcé proposé par les cellules «Cap vers l'entreprise» et par les opérateurs privés de placement, même si quelques différences ont été mises en lumière. Ainsi, les cellules de l'ANPE proposaient aux demandeurs d'emploi davantage d'offres d'emploi que les OPP, tandis que ces derniers se concentraient davantage sur l'aide à la recherche d'emploi. Toutefois, le cloisonnement entre l'enquête qualitative et l'évaluation quantitative rend difficile une évaluation d'impact du travail des conseillers sur le retour à l'emploi. Faute de données appropriées, l'étude de BeHAGHel et al. (2012) ne permet pas de savoir quelles sont les méthodes, chez les OPP comme dans le cadre du programme CVE, qui apportent une réelle plus-value à l'activité de reclassement. De son côté, l'évaluation qualitative ne repose pas sur le cadre d'analyse traditionnel des évaluations aléatoires, qui, sous certaines hypothèses, mène à l'identification de l'effet causal d'un dispositif. Ces limites ont alimenté la réflexion du présent travail d'évaluation.

En résumé, l'analyse de la littérature suggère qu'il n'existe pas à l'heure actuelle de travaux dont le but est d'évaluer l'impact de méthodes de travail innovantes pour les conseillers chargés de l'insertion des chômeurs. En particulier, il n'existe pas de travaux visant à mesurer l'impact d'une meilleure prospection du marché du travail de la part des conseillers, dans le but d'améliorer le taux d'arrivée des offres d'emploi pour les chômeurs. Pourtant, les études de terrain montrent que les pratiques en matière de reclassement se diversifient, particulièrement chez les opérateurs privés de placement (DivAY, 2010). L'objectif de cet article est de contribuer à combler ce manque en testant, à l'aide d'une expérimentation aléatoire, des dispositifs cherchant à améliorer l'efficacité du travail des conseillers. La question de la validité externe des résultats obtenus, et de l'opportunité de généraliser ces dispositifs est également posée. Nous présentons auparavant le contexte de l'étude, puis le cadre et les données de l'expérimentation.

\section{Contexte de l'évaluation}

\section{La mission Pôle Emploi chez Sodie}

Le cabinet Sodie ${ }^{(4)}$, branche du Groupe Alpha qui intervient sur toute la chaîne de l'emploi avec Sécafi(5)

(4) Pour plus d'informations, voir le site internet: http:// www.sodie.com/

(5) Expert auprès des comités d'entreprises, des comités d'hygiène, de sécurité et des conditions de travail et des organisations syndicales. et Sémaphores ${ }^{(6)}$, a débuté son existence, dans les années 1980, par la reconversion de la filière sidérurgique et a œuvré depuis à la revitalisation des territoires et au reclassement des personnels dans une série de secteurs et d'activités. C'est aujourd'hui une société de prestations et de conseil en ressources humaines, spécialisée dans l'accompagnement des transitions et des mobilités professionnelles.

Sodie a répondu en avril 2009 à l'appel d'offres émis par Pôle Emploi pour concourir au placement des demandeurs d'emploi avec des dispositifs d'accompagnement spécifiques s'inscrivant dans deux prestations, dirigées vers des publics distincts: la prestation «Trajectoire Emploi» (Trajectoire dans ce qui suit) destinée aux publics les plus éloignés de l'emploi (7), d'une part; la prestation «Licenciés économiques» (LIEC dans ce qui suit) destinée aux demandeurs d'emploi ayant signé une convention de reclassement personnalisée (CRP) ou un contrat de transition professionnelle (CTP), d'autre part ${ }^{(8)}$.

Outre le public ciblé, les prestations Trajectoire et LIEC se distinguaient par deux éléments : la durée de l'accompagnement proposé aux bénéficiaires (six mois pour le public Trajectoire, contre douze mois, au maximum, pour les licenciés économiques); le type de solution reconnue par Pôle Emploi comme permettant de mettre fin à la mission d'accompagnement. Seul le retour à l'emploi avec un contrat à durée indéterminée (CDI) ou un contrat de travail de six mois ou plus (contrat à durée déterminée [CDD], contrat de travail temporaire $[\mathrm{CTT}]$ ) était validé pour les bénéficiaires de la prestation Trajectoire, tandis que la possibilité de réaliser une formation longue ou de créer/reprendre une entreprise était également prise en compte pour les licenciés économiques.

Le type de solution reconnue par Pôle Emploi est important, dans la mesure où il permet à Sodie de déclencher ou non la facturation de la part variable de sa rémunération. Dans le cadre de cette mission, le processus de facturation est en effet le suivant: $50 \%$ de la facturation totale est dû lors de la prise

(6) Conseil auprès des entreprises et des territoires sur les champs suivants : développement des entreprises, formation/ économie/emploi, expertise et commissariat aux comptes, efficience et projets (politique de l'habitat, fiscalité, etc.).

(7) Les publics les plus éloignés de l'emploi sont définis par le cahier des charges comme étant des demandeurs d'emploi :

- inscrits en catégorie 1 ;

- qui ont besoin d'un appui durable à la recherche d'emploi;

- qui rencontrent des difficultés d'insertion et souhaitent obtenir un contrat durable;

- qui sont présents sur des bassins d'emploi peu dynamiques; - qui entrent sur le marché du travail pour la première fois.

(8) Au cours de la mission, Pôle Emploi ne parvenant pas, sur certains lots, à alimenter Sodie en demandeurs d'emploi afin de respecter les minima imposés par le marché, la prestation «Licenciés économiques» a été étendue aux licenciés économiques de droit commun (qui bénéficient du régime général d'accompagnement et d'indemnisation chômage). 
en charge du bénéficiaire, $25 \%$ lors du reclassement (seulement $10 \%$, s'il s'agit d'une formation) et $25 \%$ pour le maintien en emploi à six mois (idem pour la création/reprise d'entreprise) ${ }^{(9)}$. La facturation des deux parties variables suppose la transmission des preuves de reclassement et de maintien en emploi à Pôle Emploi.

Lors de cet appel d'offres qui était décomposé en aires géographiques, Sodie a remporté quatorze lots, soit $22 \%$ des parts de marché : pour la prestation Trajectoire, elle a ainsi été chargée de l'Est francilien (Seine-Saint-Denis et Val-de-Marne), du Centre et du Pas-de-Calais, et pour la prestation LIEC du Nord-Pas-de-Calais (deux lots), de toute la région Île-de-France hors Paris (soit trois lots), de Rhône-Alpes (deux lots), du Poitou-Charentes, de la Bourgogne, la Bretagne et la Lorraine.

Ce marché, d'une durée de deux ans à compter de septembre 2009, a permis à Sodie d'accompagner plus de 45000 bénéficiaires de la prestation LIEC et près de 20000 dans le cadre de la prestation Trajectoire. L'ampleur de la mission a contraint le cabinet à ouvrir 110 sites dans les régions concernées et à recruter une centaine de nouveaux salariés afin d'assurer, dans le cadre du cahier des charges imposé par Pôle Emploi, l'accompagnement des bénéficiaires qui leur étaient adressés.

L'ampleur de cette mission, la volonté d'innover ainsi que les apports et les limites de l'évaluation OPP/CVE évoqués plus haut ont conduit la direction de Sodie à s'engager dans une démarche autonome d'analyse et d'évaluation. L'objectif était de développer des outils permettant aux conseillers intervenant auprès des bénéficiaires (appelés conseillers ressources humaines - RH) de mesurer la portée des actions engagées et d'améliorer leurs méthodes de travail. Ainsi, un "Groupe d'analyse et d'évaluation », constitué de ressources propres au Groupe Alpha dont Sodie fait partie a été mis en place : il comptait des membres de la direction et des opérationnels de Sodie (directrice de projet, responsable de projet et conseiller RH), des membres du Centre études et prospective du groupe, la responsable de la direction des systèmes d'information chargée du déploiement de la base de données des candidats et des offres d'emploi, ainsi que des universitaires. Au terme de l'évaluation par Sodie de la mission Pôle Emploi, des conséquences opérationnelles ont été tirées, qui sont détaillées plus loin dans cet article.

(9) Ce type de rémunération variable constitue désormais la norme en matière d'externalisation des prestations de placement (FERRACCI, 2007). Il vise à inciter les opérateurs privés à investir dans des prestations efficaces, mais peut également donner lieu à des comportements de sélection : il arrive ainsi que l'essentiel des efforts des conseillers soit concentré sur les demandeurs d'emploi en apparence les moins éloignés du marché du travail.

\section{Profilage, expérimentation, enquête qualitative : les trois volets de l'évaluation de la mission Pôle Emploi chez Sodie}

L'évaluation de la mission Pôle Emploi menée au sein de Sodie a reposé sur trois démarches distinctes ${ }^{(10)}$. En premier lieu, les caractéristiques individuelles et les parcours professionnels des demandeurs d'emploi accompagnés ont été observés et analysés, afin de mieux cerner les facteurs explicatifs du retour à l'emploi et de mettre en place un système de profilage. Ce dernier consistait à estimer, pour chaque individu, la probabilité ex ante de retour à l'emploi compte tenu de ses caractéristiques observables. Le profilage visait à fournir un outil d'aide à la décision aux conseillers. En complétant leur propre jugement par l'analyse systématique des caractéristiques des bénéficiaires, il devait leur permettre d'adapter les méthodologies proposées aux besoins de chacun des demandeurs d'emploi de leur portefeuille. Élément important, l'information issue du profilage a été fournie de façon aléatoire aux conseillers des sites tirés au sort dans un des deux dispositifs évalués (voir infra, paragraphe intitulé «Description des dispositifs évalués ») ${ }^{(11)}$.

Parallèlement au profilage, deux études ont été menées: une enquête de terrain de nature qualitative (voir encadré) et l'expérimentation contrôlée de plusieurs dispositifs d'accompagnement renforcé. Ces deux études ont été conduites en étroite collaboration, l'enquête qualitative permettant de mieux cerner les facteurs de blocage opérationnels liés à la mise en œuvre de l'expérimentation $^{(12)}$. Ce travail de terrain a consisté en un ensemble d'observations et d'entretiens semi-directifs sur un échantillon de sites Sodie accueillant des demandeurs d'emploi, dans le cadre des prestations Trajectoire et LIEC. Les entretiens ont été menés auprès de responsables de projet, de conseillers RH chargés de l'accompagnement et de conseillers relations entreprises (RENT) chargés de la prospection du marché du travail et de la collecte des offres d'emploi. L'objectif était, d'une part, de mieux comprendre les interactions entre le modèle de travail (les différents métiers

(10) Ces trois démarches ont été détaillées dans une lettre du Centre études et prospective du Groupe Alpha : «Un OPP sur le divan. L'évaluation des pratiques d'accompagnement et de reclassement des demandeurs d'emploi par Sodie», La lettre du $C E P, \mathrm{n}^{\circ} 9$, février 2012. Disponible en ligne à l'adresse : http:// www.groupe-alpha.com/data/document/lettre-cep-9-opp-surdivan15-02-2012.pdf; consultée le 9 septembre 2013.

(11) L'utilisation qui est faite par les conseillers de l'information que constitue le profilage est intéressante dans la mesure où elle permet d'identifier d'éventuelles démarches d' 'écrémage» consistant à accorder le plus d'attention aux demandeurs d'emploi les moins éloignés du marché du travail dans le but d'accroître la facturation. La partie consacrée aux résultats de l'expérimentation revient sur ce point.

(12) Voir infra, paragraphe intitulé «Difficultés opérationnelles et ajustements réalisés ». 
présents chez Sodie, la répartition des tâches entre ces différents métiers, notamment entre conseillers RH et RENT, leurs liens hiérarchiques, leur degré d'autonomie) et le modèle économique de Sodie (facturation, implantation des sites, choix de rationalisation, etc.), et la manière dont ces modèles ont évolué avec la mission Pôle Emploi. D'autre part, il s'agissait d'analyser les conséquences de ces modèles sur les performances en matière de reclassement des bénéficiaires.

\section{Encadré}

\section{Méthodologie de l'enquête de terrain}

L'étude qualitative a été réalisée sur une période de dix mois, entre juin 2010 et mars 2011. Elle s'appuie sur trente-cinq entretiens semi-directifs menés auprès des collaborateurs de Sodie :

- conseillers $\mathrm{RH}$;

- conseillers RENT;

- coordinateurs RENT;

- responsables de projet;

- membres de la direction.

Dans ce volet qualitatif, le choix des personnes rencontrées devait permettre de mettre en évidence des récurrences dans les situations de travail observées. Toutes les régions concernées par la mission ont été visitées. Dans chaque région, un site a été sélectionné en fonction de différents critères, de façon à intégrer dans l'enquête la diversité des paramètres suivants :

- la prestation proposée sur le site (Trajectoire Emploi, Licenciés économiques - CTP ou CRP -, site mixte accueillant les deux prestations);

- le dynamisme du territoire (bassin d'emploi dynamique versus sinistré(1));

- la taille du site (site accueillant un nombre limité versus important de demandeurs d'emploi);

- l'implantation dans les locaux de Pôle Emploi.

L'ensemble de ces entretiens s'est appuyé sur un guide et chacun a duré en moyenne $1 \mathrm{~h} 30$. Tous ont fait l'objet d'une retranscription intégrale.

Cette étude a été scindée en deux étapes : - une phase exploratoire a été conduite afin de valider le guide d'entretien (trois sites visités et une dizaine d'entretiens réalisés en juin 2010);

- la phase de généralisation s'est déroulée entre septembre 2010 et mars 2011 et a donné lieu à la réalisation de vingt-cinq autres entretiens.

(1) La dichotomie bassin d'emploi dynamique versus bassin sinistré a été élaborée à partir des diagnostics de territoire réalisés par le service études et diagnostics de Sodie et élaborés à partir de données Insee (Institut national de la statistique et des études économiques) et Pôle Emploi notamment, telles que l'enquête «Besoins en main-d'œuvre».

La seconde évaluation mise en place a pris la forme d'une expérimentation contrôlée de plusieurs dispositifs visant à améliorer le reclassement des bénéficiaires. Cette évaluation fait l'objet des parties suivantes.

\section{L'expérimentation de dispositifs de reclassement innovants}

\section{Description des dispositifs évalués}

La démarche du groupe d'analyse et d'évaluation mis en place par Sodie a consisté à tester, lors d'une première phase préexpérimentale de six mois menée au cours du premier semestre 2010, trois dispositifs sur un échantillon réduit de sites. Ces trois dispositifs ont été élaborés en confrontant, au sein du groupe d'analyse et d'évaluation, les réflexions d'intervenants universitaires et de responsables du reclassement chez Sodie. La phase préexpérimentale a permis de faire remonter les difficultés opérationnelles liées à la mise en œuvre de chacun des dispositifs, et de les corriger dans une certaine mesure ${ }^{(13)}$. Les dispositifs comprenaient la mise en place d' « engagements mensuels de reclassement» (dispositif PARI), l'augmentation des moyens dédiés à la relation entreprises (pôle chargé de collecter des offres d'emploi et de positionner les demandeurs sur ces offres; dispositif RENT) et la publication mensuelle de la performance individuelle des conseillers $\mathrm{RH}$ (dispositif RANK).

Le dispositif RANK consistait à classer, pour chaque lot, les conseillers RH en fonction de leur performance individuelle en matière de reclassement. Ces résultats étaient ensuite communiqués mensuellement à l'ensemble des conseillers du lot. Aucune sanction ou prime supplémentaire n'était associée à la publication de ce classement. Il s'agissait de motiver les conseillers en confrontant leurs résultats et de favoriser, au sein d'un même collectif de travail, la coopération et l'échange de bonnes pratiques méthodologiques entre les plus performants et ceux qui rencontraient davantage de difficultés. Ce dispositif, particulièrement plébiscité par la direction de Sodie qui souhaitait inciter ses conseillers à la performance, n'a finalement pas été conservé lors de la phase expérimentale puisqu'il a été généralisé à l'ensemble de la société immédiatement après la préexpérimentation du fait de son coût quasi nul. Seuls les dispositifs PARI et RENT ont donc été testés en phase expérimentale.

Les conseillers concernés par le dispositif PARI devaient s'engager mensuellement à reclasser un certain nombre de demandeurs d'emploi de leur portefeuille (cinq bénéficiaires ou $10 \%$ des candidats de leur portefeuille), demandeurs nommément identifiés de façon préalable. En pratique, chaque début de mois, les conseillers concernés indiquaient sur le logiciel de suivi des candidats ceux

(13) Voir infra, paragraphe intitulé «Difficultés opérationnelles et ajustement réalisés ». 
qu'ils souhaitaient sélectionner au titre des engagements mensuels de reclassement. Ce dispositif résultait du constat, dressé par les responsables opérationnels des sites lors de la phase exploratoire de l'enquête qualitative, selon lequel les conseillers auraient des difficultés à organiser leur temps, et consacraient parfois trop d'efforts à des demandeurs d'emploi qui étaient relativement autonomes dans leur recherche. La mise en place du dispositif PARI devait leur permettre, chaque mois, de prendre le temps de passer en revue l'intégralité de leur portefeuille et d'identifier les bénéficiaires pour lesquels leur intervention accrue serait $a$ priori plus efficace que pour d'autres. Une fois ces bénéficiaires identifiés, avec l'aide de leur responsable de projet, les conseillers devaient déterminer leur planning et les actions à mener pour répondre à leurs besoins en accompagnement, tout en ne négligeant pas les autres demandeurs d'emploi de leur portefeuille. La personne responsable de la coordination de l'expérimentation était chargée de recenser et de centraliser les engagements de reclassement de l'ensemble des sites, et de relancer, le cas échéant, les conseillers qui n'auraient pas défini leurs engagements mensuels à temps. Le dispositif PARI se présentait ainsi comme une aide méthodologique pour les conseillers, visant à améliorer leur efficacité en les incitant à mieux organiser leur temps ${ }^{(14)}$.

Le second dispositif testé correspondait à une augmentation des moyens du pôle relations entreprises de Sodie (RENT) dédié à la collecte des offres d'emploi. Quatre assistantes RENT ont été recrutées pour les besoins et la durée de l'expérimentation, et ont eu pour mission d'alimenter en offres exclusivement les sites bénéficiaires du dispositif après tirage au sort. Ces assistantes étaient chargées de prospecter le marché ouvert (15), en identifiant, pour un portefeuille limité de demandeurs d'emploi, les offres d'emploi pertinentes issues des principales sources disponibles. De plus, elles devaient également assurer l'envoi des CV et le suivi de chacune des candidatures transmises aux employeurs. Au-delà de la prospection, le dispositif RENT comporte donc une dimension relative au positionnement des offres.

(14) Même si les conseillers y ont été sensibilisés afin de le limiter, ce dispositif peut engendrer un «écrémage» de certains demandeurs d'emploi, considérés comme non reclassables et laissés de côté au profit de ceux sélectionnés au titre des engagements mensuels de reclassement. Un tel risque est néanmoins limité par le fait que, comme le dispositif RANK, le dispositif PARI n'est couplé à aucune sanction ou prime complémentaire. L'analyse des résultats montre par ailleurs que les individus du portefeuille les plus éloignés du marché du travail au regard de leurs caractéristiques observables n'ont statistiquement pas été pénalisés par la mise en œuvre du dispositif PARI (voir infra, paragraphe intitulé «Résultats par sous-populations»).

(15) Le marché ouvert du travail concerne tous les postes à pourvoir qui font l'objet d'une annonce, en opposition au marché caché.
Selon la logique du dispositif, l'augmentation des moyens et l'amélioration du processus de rapprochement entre offre et demande d'emploi devraient accroître les performances en matière de reclassement ${ }^{(16)}$.

\section{Données utilisées et variables de résultat}

Pour la constitution des groupes tests et de contrôle, comme pour l'évaluation des dispositifs, les données utilisées ont toutes été issues du logiciel interne de gestion des candidats, nommé «RHapSodie». L'utilisation d'une base de données interne à un cabinet de reclassement constitue l'une des originalités de la présente expérimentation. D'une part, le logiciel RHapSodie permettait aux conseillers de saisir et de visualiser l'ensemble des caractéristiques individuelles des demandeurs d'emploi accompagnés : niveau de qualification, parcours professionnel antérieur, projet professionnel, etc. D'autre part, les offres d'emploi collectées par le pôle relation entreprises étaient également entrées dans le logiciel interne. Elles étaient à la disposition des conseillers RH qui pouvaient réaliser directement l'appariement entre une offre d'emploi et un de leurs bénéficiaires. Le suivi de chaque candidature était, de plus, disponible et visualisable par les conseillers sur RHapSodie : données relatives au parcours d'accompagnement et au reclassement du demandeur d'emploi, type de prestation proposée, rendez-vous ou ateliers effectués, date de sortie du dispositif, type de reclassement (emploi, formation, création/reprise d'entreprise) ${ }^{(17)}$. Un travail de sensibilisation à l'importance du remplissage des données contenues dans le logiciel a été mené auprès de l'ensemble des conseillers RH.

La variable de résultat retenue est la sortie positive durable (cf. supra), un certain temps après

(16) Chez Sodie le pôle relations entreprises est organisé régionalement autour de quatre métiers :

- le conseiller RENT : un par lot dans le cadre de la mission Pôle Emploi. Il prospecte le marché caché de l'emploi en allant rencontrer les entreprises implantées sur son territoire et ayant des postes à pourvoir;

- l'assistante RENT : également une par lot. Elle peut être chargée de saisir informatiquement les offres d'emploi collectées par le consultant RENT. Elle peut également assurer l'appariement entre offre et demande en envoyant les CV transmis par les conseillers RH aux recruteurs et en suivant les candidatures;

- les téléprospecteurs : ils appellent les entreprises pour collecter des offres d'emploi. L'effort de téléprospection est variable en fonction des lots;

- le coordinateur RENT pilote, dans chaque région, le pôle relation entreprises.

Ainsi, tandis que traditionnellement le pôle relations entreprises intervenait sur le marché caché, les assistantes RENT recrutées dans le cadre de l'expérimentation prospectaient uniquement le marché ouvert, via la consultation de sites dédiés.

(17) De ce point de vue, les données relatives à la mission Pôle Emploi offrent des perspectives pour des recherches ultérieures plus spécifiques que la présente expérimentation. Voir sur ce point la conclusion de l'article. 
l'adhésion à l'une des deux prestations d'accompagnement (Trajectoire ou LIEC). On entend par sortie positive durable toutes les sorties vers un emploi en CDI ou ayant une durée contractuelle d'au moins six mois, quel que soit le type de contrat (CDD ou contrat d'intérim). Sont également comptabilisées pour la prestation LIEC les formations longues, de plus de six mois et de 150 heures, ou la sortie pour création ou reprise d'entreprise. Élément important, dans la base de données RHapSodie, les sorties vers l'emploi ou la formation ne donnent lieu à facturation que lorsqu'une preuve formelle est apportée par le demandeur d'emploi (contrat de travail, attestation d'entrée en formation par exemple). Ce mode de validation des sorties comporte une part d'aléas, certains demandeurs d'emploi omettant parfois de renvoyer à Sodie les documents attestant leur nouvelle situation. De ce point de vue, les taux de sortie obtenus doivent être considérés avec prudence, en particulier lorsqu'ils sont comparés à des études établissant le retour à l'emploi de façon déclarative et orale, comme dans le cas de l'évaluation OPP/CVE (BeHAGHEL et al., 2012).

\section{Tirage aléatoire et comparaison des caractéristiques moyennes des bénéficiaires}

Le tirage au sort a porté sur 101 sites Sodie ouverts dans le cadre de la mission Pôle Emploi, répartis dans huit régions et accueillant des bénéficiaires des deux prestations, Trajectoire et LIEC. Les trois sites sélectionnés pour le test des dispositifs en phase préexpérimentale ont été exclus du tirage au sort. Ce dernier a porté sur les sites, et non sur les bénéficiaires eux-mêmes, pour des questions à la fois déontologiques et pratiques. Il semblait en effet délicat d'accueillir sur un même site des demandeurs d'emploi bénéficiant d'un dispositif complémentaire destiné à améliorer leurs perspectives de sortie, et d'autres n'en bénéficiant pas. En outre, la nature même des dispositifs testés rendait difficile un traitement différencié des demandeurs d'emploi au sein d'un même site. L'expérimentation a porté sur une période de dix mois, entre septembre 2010 et juin 2011. Les sites ont été répartis aléatoirement en trois groupes : les deux dispositifs testés (PARI et RENT) et le groupe de contrôle. Un tirage au sort a également été effectué avant d'allouer à chacune des assistantes de la plateforme de positionnement RENT un certain nombre de sites sur lesquels travailler. Procéder à un tirage sur les sites et non sur les individus a notamment pour conséquence que sur les sites traités, deux types de demandeurs d'emploi sont observés : ceux qui étaient déjà inscrits au moment du début de l'expérimentation (le stock) et ceux qui sont entrés en chômage après cette date (le flux). Dans la mesure où ces deux sous-populations ne sont pas comparables au regard de leurs durées de chômage potentielles, des effets distincts ont été estimés pour la population du stock et celle $\mathrm{du}$ flux. Par ailleurs, afin d'assurer une certaine homogénéité dans la taille des sites au sein des différents groupes, une stratification des sites par taille a été réalisée préalablement au tirage au sort. La distribution des sites par taille a été scindée en trente-quatre paquets de trois sites de taille équivalente, et le tirage au sort pour alimenter les groupes (PARI, RENT et de contrôle) a été réalisé au sein de chacun de ces paquets.

L'objectif du tirage au sort était de constituer au sein des deux groupes Trajectoire et LIEC des populations d'individus similaires au regard de leurs caractéristiques individuelles, et ne différant que par leur statut vis-à-vis des dispositifs d'accompagnement dont ils bénéficiaient. Les tableaux 1 et 2 proposent des éléments de statistique descriptive suggérant que cet objectif est atteint, à la fois pour la population du stock et la population du flux. Pour chacune des deux prestations, les trois premières colonnes présentent les moyennes d'un ensemble de caractéristiques individuelles au sein des groupes RENT, PARI et du groupe de contrôle. Les populations de ces trois colonnes ayant été déterminées au hasard, on y vérifie que leurs caractéristiques moyennes sont semblables. Certes, des différences y apparaissent ponctuellement. Elles sont néanmoins petites et rarement significatives, comme le montrent les colonnes suivantes $\mathrm{du}$ tableau, qui contiennent des étoiles indiquant les différences statistiquement significatives. Les groupes expérimentaux ont donc des caractéristiques observables comparables. Mais on peut aller plus loin : si l'affectation aléatoire a eu pour effet de rendre les groupes comparables au regard de leurs dimensions observables (telles que l'âge ou le diplôme du demandeur d'emploi), on est fondé à affirmer qu'ils le sont aussi au regard de dimensions inobservables et susceptibles d'avoir un effet sur le retour à l'emploi (telles que la motivation ou l'étendue du réseau social).

Au-delà de la validation du cadre expérimental que permet la comparaison des moyennes, les tableaux 1 et 2 apportent des indications sur les caractéristiques respectives des populations Trajectoire et LIEC. Logiquement, les individus bénéficiant de la prestation Trajectoire présentent des caractéristiques rendant moins probable leur retour à l'emploi. Ces caractéristiques sont résumées dans un score de propension individuel représentant la probabilité d'être en emploi un an après l'inscription au chômage. Le score est obtenu grâce au modèle de profilage développé par Sodie dans le cadre de l'évaluation de la mission Pôle Emploi. Conformément à l'intuition, le score moyen est moins élevé (autour de 0,15 ) dans les groupes de la prestation Trajectoire que dans le groupe des licenciés économiques (autour de 0,35). 
Tableau 1 : Caractéristiques individuelles au sein des groupes expérimentaux (moyennes) - Population du stock

\begin{tabular}{|c|c|c|c|c|c|c|c|c|c|c|}
\hline \multirow[t]{2}{*}{ Population des bénéficiaires } & \multicolumn{5}{|c|}{ Trajectoire } & \multicolumn{5}{|c|}{ LIEC } \\
\hline & $\begin{array}{c}\text { RENT } \\
\text { (1) }\end{array}$ & $\begin{array}{l}\text { PARI } \\
\text { (2) }\end{array}$ & $\begin{array}{c}\text { Contrôle } \\
\text { (3) }\end{array}$ & (1) - (3) & (2) - (3) & $\begin{array}{c}\text { RENT } \\
\text { (1) }\end{array}$ & $\begin{array}{l}\text { PARI } \\
\text { (2) }\end{array}$ & $\begin{array}{c}\text { Contrôle } \\
\text { (3) }\end{array}$ & (1) - (3) & (2) - (3) \\
\hline Femme & 0,46 & 0,46 & 0,45 & & & 0,41 & 0,40 & 0,41 & & $*$ \\
\hline$\hat{\text { Âge }}<35$ ans & 0,33 & 0,34 & 0,38 & $*$ & * & 0,39 & 0,38 & 0,39 & & \\
\hline Célibataire & 0,38 & 0,37 & 0,40 & & * & 0,21 & 0,21 & 0,22 & & \\
\hline Français & 0,79 & 0,84 & 0,85 & $*$ & & 0,94 & 0,93 & 0,93 & & \\
\hline Sans diplôme & 0,23 & 0,24 & 0,23 & & & 0,09 & 0,10 & 0,08 & & \\
\hline Permis de conduire & 0,52 & 0,55 & 0,56 & * & & 0,82 & 0,80 & 0,81 & & \\
\hline CSP : employé & 0,39 & 0,41 & 0,41 & $*$ & & 0,45 & 0,43 & 0,44 & & \\
\hline Dernière période de chômage $<100$ jours & 0,28 & 0,30 & 0,31 & * & & 0,20 & 0,22 & 0,21 & * & \\
\hline Salaire demandé $=$ Smic & 0,32 & 0,33 & 0,34 & & & 0,10 & 0,11 & 0,11 & & \\
\hline Dernier contrat : CDI & 0,24 & 0,30 & 0,28 & * & & 0,81 & 0,80 & 0,84 & & $*$ \\
\hline Reconversion $^{\circ}$ & 0,55 & 0,50 & 0,51 & $*$ & * & 0,49 & 0,48 & 0,48 & * & \\
\hline Score individuel $^{\circ \circ}$ & 0,13 & 0,12 & 0,13 & & & 0,32 & 0,31 & 0,32 & & \\
\hline Taille des sites ${ }^{\circ \circ}$ & 398 & 441 & 481 & $* *$ & $* *$ & 398 & 441 & 481 & $* *$ & $* *$ \\
\hline Nombre d'individus & 3515 & 1576 & 5382 & & & 3446 & 4963 & 4739 & & \\
\hline
\end{tabular}

Lecture : ***,*** : significatif au seuil de $10 \%, 5 \%$ et $1 \%$.

${ }^{\circ}$ La variable reconversion vaut 1 si le code ROME (Répertoire opérationnel des métiers et des emplois) du métier recherché est différent de celui de l'emploi précédent.

${ }^{\circ}$ Le score est calculé en régressant le statut d'emploi après un an sur un ensemble de caractéristiques individuelles.

${ }^{\circ}$ La taille des sites correspond au nombre moyen d'individus dans chaque groupe de sites, toutes prestations confondues. On contrôle ainsi que les écarts entre les différents groupes en termes de taille des sites sont trop faibles pour générer des différences d'organisation du travail entre petits et grands sites.

Source : Sodie.

Tableau 2 : Caractéristiques individuelles au sein des groupes expérimentaux (moyennes) - Population du flux

\begin{tabular}{|c|c|c|c|c|c|c|c|c|c|c|}
\hline \multirow[t]{2}{*}{ Population des bénéficiaires } & \multicolumn{5}{|c|}{ Trajectoire } & \multicolumn{5}{|c|}{ LIEC } \\
\hline & RENT & PARI & Contrôle & (1) - (3) & (2) - (3) & RENT & PARI & Contrôle & (1) - (3) & (2) - (3) \\
\hline & (1) & (2) & (3) & & & (1) & (2) & (3) & & \\
\hline Femme & 0,45 & 0,45 & 0,44 & & & 0,44 & 0,42 & 0,42 & & \\
\hline$\hat{\text { Âge }}<35$ ans & 0,41 & 0,40 & 0,38 & $*$ & & 0,42 & 0,42 & 0,41 & & \\
\hline Célibataire & 0,39 & 0,36 & 0,38 & & * & 0,21 & 0,21 & 0,22 & & \\
\hline Français & 0,90 & 0,91 & 0,93 & $*$ & & 0,95 & 0,94 & 0,94 & & \\
\hline Permis de conduire & 0,65 & 0,64 & 0,62 & $*$ & & 0,85 & 0,86 & 0,85 & & \\
\hline Sans diplôme & 0,11 & 0,10 & 0,10 & & & 0,10 & 0,08 & 0,08 & $*$ & \\
\hline Employé & 0,43 & 0,45 & 0,46 & $*$ & & 0,45 & 0,43 & 0,44 & & \\
\hline Dernière période de chômage $<100$ jours & 0,36 & 0,35 & 0,33 & $*$ & & 0,21 & 0,20 & 0,19 & * & \\
\hline Salaire demandé $=$ Smic & 0,33 & 0,34 & 0,32 & & * & 0,08 & 0,08 & 0,09 & & \\
\hline Dernier contrat : CDI & 0,31 & 0,32 & 0,33 & * & & 0,93 & 0,92 & 0,92 & & * \\
\hline Reconversion $^{\circ}$ & 0,48 & 0,50 & 0,51 & & & 0,50 & 0,49 & 0,48 & * & \\
\hline Score individuel ${ }^{\circ \circ}$ & 0,18 & 0,19 & 0,19 & & & 0,37 & 0,37 & 0,37 & & \\
\hline Taille des sites ${ }^{\circ \circ \circ}$ & 283 & 380 & 402 & $* *$ & & 283 & 380 & 402 & $* *$ & \\
\hline Nombre d'individus & 1994 & 1719 & 3112 & & & 2987 & 3888 & 4244 & & \\
\hline
\end{tabular}

Lecture : *,*, *** : significatif au seuil de $10 \%, 5 \%$ et $1 \%$.

'La variable reconversion vaut 1 si le code ROME du métier recherché est différent de celui de l'emploi précédent.

${ }^{\circ}$ Le score est calculé en régressant le statut d'emploi après un an sur un ensemble de caractéristiques individuelles.

${ }^{\circ \circ}$ La taille des sites correspond au nombre moyen d'individus dans chaque groupe de sites, toutes prestations confondues.

Source : Sodie.

\section{Difficultés opérationnelles et ajustement réalisés}

L'expérimentation s'est heurtée à plusieurs types de difficultés opérationnelles et des ajustements ont dû être opérés par rapport au protocole scientifique et aux méthodologies initialement prévus. Tout d'abord, concernant le processus aléatoire, certains sites ont dû être exclus de l'expérimentation après le tirage au sort initialement réalisé. Trois sont ainsi sortis du protocole parce qu'ils ont décidé de mener, pendant la durée de l'expérimentation, une démarche similaire au dispositif RENT alors même qu'ils se trouvaient dans le groupe de contrôle ou le groupe PARI.

Des difficultés sont également apparues lors de la mise en œuvre opérationnelle des deux 
dispositifs expérimentés. Ainsi, dans le cadre de l'augmentation des moyens humains du pôle relations entreprises, les quatre assistantes devant être embauchées n'ont pu l'être qu'en octobre 2010, soit un mois après le lancement de l'expérimentation, et la phase d'apprentissage pour la prospection et le positionnement des offres a été plus ou moins longue selon les assistantes. De plus, compte tenu du turn-over de l'équipe, sur certaines périodes (notamment en mai-juin 2011), la plateforme n'a été composée que de trois assistantes, au lieu de quatre. Pendant cette phase, les sites alloués à l'assistante manquante ont été réaffectés aux autres assistantes.

Une autre difficulté rencontrée dans le cadre du dispositif RENT a nécessité le recentrage vers certains demandeurs d'emploi à traiter en priorité. En effet, au début de l'expérimentation de la plateforme de positionnement, les assistantes étaient chargées de chercher des offres d'emploi pour l'ensemble des candidats faisant l'objet d'un accompagnement sur les sites de leur portefeuille et disposant déjà d'un CV. Étant donné le nombre important de demandeurs d'emploi à traiter et des refus fréquents de répondre aux offres de la part de certains candidats ${ }^{(18)}$, il a été décidé de concentrer le travail de ces quatre assistantes relations entreprises sur le public le plus éloigné de l'emploi (pour la prestation Trajectoire) et sur les candidats en fin de CTP/CRP, plus susceptibles d'accepter. Une fois ce recentrage effectué, les refus de positionnement ont effectivement baissé, rendant plus efficace le travail de la plateforme.

Le dispositif des engagements mensuels de reclassement a également connu une évolution notable en cours d'expérimentation, avec la communication aux conseillers d'informations relatives au profilage des demandeurs d'emploi en février 2011. Avec cet outil d'aide à la décision, il s'agissait d'aider les conseillers dans leur choix de candidats à sélectionner mais également d'observer leur comportement de sélection. Plus précisément, la transmission des scores de profilage s'est faite en deux temps afin de pouvoir mesurer l'évolution des comportements : en février et mars 2011, les données ont été remises aux conseillers intervenant sur la moitié des sites bénéficiant du PARI; et ce n'est qu'à partir d'avril 2011 qu'elles ont été fournies à l'ensemble des consultants concernés par le PARI.

(18) Chez les licenciés économiques en début de CRP/CTP, ces refus de candidater sur les offres soumises par l'assistante RENT peuvent être dus au fait que l'indemnisation chômage reçue est élevée et correspond à $80 \%$ du dernier salaire brut, ce qui peut expliquer, qu'en début de période de chômage, certains licenciés économiques soient dans une phase d'attente.

\section{Résultats}

\section{Impact du traitement et externalités au sein des sites expérimentaux}

La mesure de l'impact de chaque dispositif est réalisée sur l'ensemble des individus des sites traités, afin de prendre en compte les externalités potentielles, positives comme négatives. En effet, le fait pour un demandeur d'emploi de se situer sur un site RENT n'implique pas nécessairement qu'il bénéficiera à titre individuel de l'appui de la plateforme de positionnement. En revanche, il est possible que sur les sites RENT, l'information fournie à certains demandeurs d'emploi par la plateforme circule et bénéficie aux individus dont le CV n'avait pas été envoyé vers les annonces identifiées par les assistantes de la plateforme. Ceci constituerait une externalité positive, mais il est à l'inverse possible d'envisager des effets indirects négatifs, notamment dans le cadre du dispositif PARI qui voit les conseillers sélectionner plusieurs demandeurs d'emploi de leur portefeuille pour concentrer, de façon temporaire, leurs efforts sur eux. Il est possible que ceci s'accompagne d'une moindre attention à l'égard des demandeurs du site qui n'auraient pas été retenus par les conseillers dans le cadre du PARI. Ce type d'externalités potentielles justifie de mesurer l'impact des deux dispositifs sur l'ensemble des demandeurs d'emplois des sites concernés, que ceux-ci aient été choisis ou pas dans le cadre des engagements mensuels ou par la plateforme de positionnement.

L'évaluation est effectuée pour les deux prestations Trajectoire et LIEC, et pour deux types de population: les demandeurs d'emploi du stock et ceux du flux. Les demandeurs d'emploi du stock sont ceux ayant adhéré à un des deux dispositifs avant le lancement de l'expérimentation contrôlée, en septembre 2010. Quant à la population du flux, il s'agit des demandeurs d'emploi qui ont adhéré en cours d'expérimentation, c'est-à-dire entre septembre 2010 et juin 2011. Analyser de manière séparée l'effet des dispositifs sur le reclassement durable de la population du stock d'une part, et du flux d'autre part, permet de mesurer l'efficacité des dispositifs sur des demandeurs d'emploi qui en bénéficient dès le début de leur épisode de chômage (par exemple : les licenciés économiques du flux) ou sur des demandeurs d'emploi bénéficiant déjà préalablement de l'expérimentation d'un accompagnement (par exemple, les candidats de la prestation Trajectoire du stock).

\section{Résultats par sous-populations}

Le tableau 3 présente l'impact des dispositifs RENT et PARI sur la probabilité de sortir vers l'emploi ou la formation pour les bénéficiaires de la prestation LIEC. Les chiffres entre parenthèses indiquent le taux de sortie moyen pour la population 
considérée ${ }^{(19)}$. Les paramètres estimés représentent ainsi, en points de pourcentage, les augmentations ou diminutions dans le taux de sortie que l'on peut attribuer spécifiquement au dispositif. Quel que soit l'horizon temporel, il apparaît que les deux dispositifs ont un impact positif sur la population du flux, cet effet étant significatif, et légèrement plus marqué, pour le dispositif PARI. Ainsi, ce dernier accroît de six points la probabilité de sortir vers l'emploi ou la formation douze mois après l'adhésion au dispositif ( +5 points pour le dispositif RENT). S'agissant de la population du stock, les résultats sont plus mitigés, puisque le dispositif RENT n'a d'effet significatif et positif qu'à 6 mois, tandis que le dispositif PARI ne montre aucun effet significatif.

\section{Tableau 3 : Impact des dispositifs sur la probabilité de sortie vers l'emploi, la création/reprise d'entreprise ou la formation. Modèle de probabilité linéaire}

\begin{tabular}{|c|c|c|c|}
\hline \multicolumn{4}{|c|}{ Prestation : LIEC } \\
\hline \multicolumn{3}{|c|}{ 3 mois après adhésion } \\
Flux (14 \%) & Stock (9 \%) \\
RENT & PARI & RENT & PARI \\
0,01 & $0,03^{*}$ & 0,01 & 0,00 \\
\hline \multicolumn{3}{|c|}{ 6 mois après adhésion } \\
Flux (22 \%) & Stock (18 \%) \\
RENT & PARI & RENT & PARI \\
$0,04^{* *}$ & $0,05^{* *}$ & $0,02^{*}$ & 0,01 \\
\hline \multicolumn{4}{|c|}{ 12 mois après adhésion } \\
Flux (31 \%) & Stock (30 \%) \\
RENT & PARI & RENT & PARI \\
$0,05^{* *}$ & $0,06^{* * *}$ & $-0,03$ & 0,01 \\
\hline
\end{tabular}

${ }^{\circ}$ Compte tenu de la variable de résultat retenue, il faut noter que la population du stock peut être hétérogène car composée d'individus dont la durée d'accompagnement peut varier entre 0 et 3 mois pour le taux de sortie à 3 mois, entre 0 et 12 mois pour le taux de sortie à 12 mois. Pour cette raison, une comparaison directe avec les résultats du flux est également difficile.

Lecture : *, **, *** : significatif à $10 \%, 5 \%$ et $1 \%$. Les écarts-types sont estimés sur la population des sites (clustering).

Pour les licenciés économiques du flux, le fait de bénéficier du dispositif RENT augmente leur probabilité de sortie durable de 5 points de $\%$ par rapport au groupe de contrôle, 12 mois après adhésion.

Source : Sodie

Le tableau 4 présente l'impact des deux dispositifs sur le taux de sortie des bénéficiaires de la prestation Trajectoire. Dans ce cadre, le dispositif RENT produit toujours des effets positifs pour la population du flux. L'élément marquant est que l'effet du PARI pour cette population est désormais

(19) Il convient de rappeler que ces taux de sortie reposent sur l'obtention par les conseillers d'éléments attestant le reclassement ou l'accès à la formation, et ne sont ainsi pas directement comparables aux taux obtenus suite à des enquêtes téléphoniques auprès des demandeurs d'emploi dans le cadre de travaux d'évaluation similaires. non significatif à 3 mois et même significativement négatif à 6 mois (- 2 points de pourcentage) ${ }^{(20)}$. Pour la population du stock, aucun des deux dispositifs n'a d'effet significatif pour les bénéficiaires de la prestation Trajectoire, quel que soit l'horizon temporel considéré. Le fait que le dispositif PARI ait un effet négatif ici peut être dû à une externalité négative, à l'image de celles récemment mises en évidence pour d'autres politiques actives du marché du travail(21). Ce dispositif peut en effet s'accompagner d'une moindre attention portée à certains demandeurs d'emploi. Il est possible que, concernant la population LIEC, composée d'individus en moyenne moins éloignés du marché du travail et sans doute plus autonomes dans leur recherche d'emploi, cette moindre attention ait moins de conséquences. En revanche, pour ceux de la population Trajectoire, le fait de n'être pas retenus dans le cadre des engagements mensuels de reclassement peut avoir un effet plus délétère, si l'on suit l'hypothèse selon laquelle ils nécessitent un accompagnement plus serré que les licenciés économiques.

Si les bénéficiaires de Trajectoire souffrent plus $\mathrm{du}$ « délaissement», rien n'indique en revanche que les conseillers choisissent prioritairement, dans le cadre de leurs engagements mensuels de reclassement, les individus les moins éloignés du marché du travail. Il semblerait que ce soit plutôt le contraire, comme en témoigne l'utilisation faite par les conseillers des sites PARI du score individuel. À partir d'avril 2011 (pour moitié à partir de février 2011), les coordinateurs de l'expérimentation ont communiqué aux conseillers PARI le score de tous les demandeurs d'emploi de leur portefeuille(22). Une telle information a modifié leurs choix d'engagements de reclassement : à partir de février 2011, le score moyen des individus figurant dans les engagements mensuels a en effet diminué globalement, mais aussi site par site. Munis du score individuel, les conseillers ont donc délibérément concentré leurs efforts sur les individus les plus éloignés du marché du travail au regard de leurs caractéristiques observables.

(20) Compte tenu du fait que la durée de la prestation Trajectoire est limitée à 6 mois, nous n'avons pas présenté l'effet sur le taux de sortie à 12 mois ici.

(21) À l'aide des données du fichier national des Assedic, FERRACCI et ses coauteurs (2013) montrent ainsi que l'augmentation du nombre de demandeurs d'emploi formés sur un marché du travail local réduit la probabilité de retour à l'emploi des individus non formés, probablement en raison d'un effet d'éviction. Un résultat similaire est établi pour les politiques d'accompagnement des chômeurs dans le cadre d'une expérimentation aléatoire (CRÉPON et al., 2013).

(22) Cette information n'était en revanche pas disponible aux conseillers des sites RENT et des sites du groupe de contrôle avant avril. 
Tableau 4 : Impact des dispositifs sur la probabilité de sortie vers l'emploi. Modèle de probabilité linéaire

\begin{tabular}{|l|c|c|c|}
\hline \multicolumn{3}{|c|}{ Prestation : Trajectoire } \\
\hline \multicolumn{3}{|c|}{3 mois après adhésion } \\
\\
Flux (11 \%) & Stock (9 \%) \\
RENT & PARI & RENT & PARI \\
$0,03 * *$ & $-0,01$ & $-0,01$ & $-0,01$ \\
\hline \multicolumn{4}{|c|}{ 6 mois après adhésion } \\
\hline \multicolumn{3}{|c|}{ Slux (16 \%) } \\
RENT & PARI & RENT & PARI \\
$0,03 * *$ & $-0,02 * *$ & $-0,01$ & $-0,02$ \\
\hline
\end{tabular}

${ }^{\circ}$ Compte tenu de la variable de résultat retenue, il faut noter que la population du stock peut être hétérogène car composée d'individus dont la durée d'accompagnement peut varier entre 0 et 3 mois pour le taux de sortie à 3 mois, entre 0 et 6 mois pour le taux de sortie à 6 mois. Pour cette raison, une comparaison directe avec les résultats du flux est également difficile.

Lecture : *,**,*** : significatif à $10 \%, 5 \%$ et $1 \%$. Les écarts-types sont estimés sur la population des sites (clustering).

Pour les bénéficiaires de la prestation Trajectoire issus du flux, le fait de bénéficier du dispositif RENT augmente leur probabilité de sortie durable de 3 points de \% par rapport au groupe de contrôle, 6 mois après adhésion.

Source : Sodie

\section{Enseignements opérationnels et généralisation potentielle des résultats}

Les résultats de l'expérimentation montrent que le dispositif RENT améliore significativement les performances des sites sur lesquels il est mis en œuvre. Ceci n'est guère surprenant dans la mesure où la combinaison d'une meilleure prospection du marché de l'emploi et d'un travail spécifique de positionnement des CV sur les offres doit mécaniquement améliorer le taux de sortie vers l'emploi. Pour autant, la généralisation d'un dispositif similaire au RENT, au sein d'un cabinet comme Sodie ou à l'échelle plus large du service public de l'emploi, pose la question du rapport coût/bénéfice. Le recrutement des assistantes, le fonctionnement de la plateforme de positionnement pendant la durée de l'expérimentation mais aussi la recherche de nouvelles assistantes pour pallier le départ de celles ayant quitté le dispositif ont engendré des coûts tels qu'ils ont dissuadé les dirigeants de Sodie de généraliser les plateformes de prospection et de positionnement à l'ensemble des sites au terme de l'expérimentation.

Cependant, il faut noter que l'évaluation, dans ses dimensions quantitative et qualitative, a conduit Sodie à faire évoluer ses pratiques en matière de collecte des offres d'emploi. Menée dans le cadre de l'enquête qualitative, une analyse plus fine de l'activité des conseillers chargés de la prospection et du positionnement des offres a révélé que l'activité de recherche des offres était perçue par les conseillers comme prenant trop de temps, au détriment de l'activité d'analyse des CV des candidats et de positionnement de ces derniers sur les offres. Dans la mesure où nombre de plateformes de recrutement en ligne collectent à l'heure actuelle des offres de façon automatisée et en grand nombre, la direction de Sodie a décidé de nouer un partenariat avec l'une d'elles pour accéder à ses offres ${ }^{(23)}$. L'objectif de cette démarche est de recentrer l'activité de l'ensemble des conseillers RENT sur la mise en regard des offres et des $\mathrm{CV}$, opération qui constitue leur véritable valeur ajoutée.

En revanche, le dispositif PARI présente un intérêt économique plus net, dès lors qu'il est correctement ciblé. En effet, ce dispositif n'engendre pas de surcoût majeur, si l'on excepte la nécessité d'un suivi des engagements mensuels de reclassement par un coordinateur chargé de relancer les conseillers n'ayant pas satisfait leurs obligations en la matière. Moyennant quoi, le succès du dispositif PARI, même s'il est limité à la population des licenciés économiques, indique qu'investir dans des méthodes de travail innovantes peut améliorer l'efficacité du placement dans des proportions similaires à celle d'un accompagnement renforcé, nécessairement plus coûteux. Ceci constitue un résultat important de cet article. Pour pallier les limites des ressources du service public de l'emploi dans un contexte budgétaire difficile, une réflexion systématique sur les méthodes de travail des conseillers devrait ainsi être envisagée, et prolongée par la généralisation des pratiques les plus performantes.

Il convient toutefois d'être prudent quant à la possible généralisation de dispositifs proches de celui du PARI. L'enquête qualitative menée par Sodie a mis en évidence les tensions qu'exerce sur l'activité des conseillers la conjonction d'une obligation de résultats et de l'accroissement du flux des demandeurs d'emplois pris en charge. Cette contrainte est partagée par les opérateurs privés de placement, mais aussi, dans une certaine mesure, par les conseillers de Pôle Emploi. Dans ce contexte, il peut apparaître délicat de piloter, sur des sites décentralisés, des dispositifs comportant une dimension méthodologique importante. Dans le cadre du PARI, les conseillers ont parfois perçu de manière négative l'obligation des choix des engagements mensuels et leur suivi. Ainsi, plutôt qu'une généralisation à marche forcée, l'analyse des difficultés opérationnelles rencontrées dans le cadre de l'expérimentation milite en faveur d'un échange constant et organisé de bonnes pratiques. De ce point de vue, l'expérimentation a mis en évidence des effets différenciés des deux dispositifs sur les populations de flux et de stock, dont les durées de chômage potentielles diffèrent. Un ciblage des dispositifs sur les sous-populations les plus aptes à en tirer profit, en l'occurrence les licenciés économiques pour le PARI, permettrait d'améliorer encore l'efficacité de l'accompagnement.

(23) Ce partenariat a été signé le 10 décembre 2012 avec la plateforme Qapa.fr. 


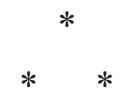

Cet article présente le cadre, la méthode et les résultats d'une expérimentation aléatoire visant à tester, au sein d'un cabinet privé de placement, l'effet sur le taux de sortie du chômage de deux dispositifs de reclassement innovants. Le premier (RENT) a pour objectif d'intensifier la prospection du marché du travail, afin d'accroître le nombre d'offres soumises aux demandeurs d'emploi accompagnés. Ses effets sur le retour à l'emploi sont globalement positifs, mais son coût important semble constituer un obstacle à sa généralisation. Le second dispositif (PARI) consiste à fournir aux consultants une aide méthodologique, en les incitant à concentrer temporairement leurs efforts sur un nombre limité de demandeurs d'emplois. Ce dispositif, dont le coût est bien moindre que celui du RENT, produit également des effets positifs, mais sur la seule population des individus ayant fait l'objet d'un licenciement économique.

L'article contribue à alimenter la littérature empirique sur l'efficacité des dispositifs de reclassement des chômeurs en adoptant un angle relativement nouveau : celui des conseillers chargés du placement, et non celui des demandeurs d'emploi. Les résultats obtenus montrent que fournir un appui aux conseillers peut s'avérer tout aussi efficace que renforcer les dispositifs d'accompagnement.

Dans un futur proche, des travaux pourraient approfondir ces résultats dans deux directions : d'une part, en produisant une véritable analyse coût/bénéfice de chacun des dispositifs tenant compte des recettes qu'ils génèrent dans le cadre d'une rémunération des opérateurs privés de placement comportant une part variable; d'autre part, en évaluant spécifiquement l'ampleur des externalités engendrées par les dispositifs (effets positifs de diffusion de l'information, ou négatifs de délaissement de certains demandeurs d'emploi) au moyen de données collectées lors de l'expérimentation et non encore utilisées jusqu'à présent. En particulier, le dispositif PARI devrait faire l'objet d'investigations plus poussées afin de mieux comprendre les modifications de comportement qu'il entraîne chez les conseillers, notamment lorsque ceux-ci disposent d'une information supplémentaire sous forme de score de profilage.

\section{Bibliographie}

Behaghel L., Crépon B., Gurgand M. (2012), « Private and public provision of counseling to job-seekers: evidence from a large controlled experiment », IZA discussion paper, $\mathrm{n}^{\circ} 6518$.

Blundell R., Costa Dias M., Meghir C., Van Reenen J. (2004), « Evaluating the employment impact of a mandatory job search assistance program », Journal of the European economic association, vol. 2, $\mathrm{n}^{\circ} 4$, pp. 569-606.

Capelier T., Mizrahi R. (2008), «L'évaluation qualitative de la mise en œuvre des expérimentations », L'accompagnement renforcé des demandeurs d'emploi, $\mathrm{n}^{\circ} 2$, publication conjointe ANPE-Dares-Unedic.

Card D., Kluve J., Weber A. (2010), « Active labour market policy evaluations: a meta-analysis », The economic journal, vol. 120, $\mathrm{n}^{\circ}$ 548, pp. F452-F477.

Crépon B., Dejemeppe M., Gurgand M. (2005), « Counseling the unemployed: does it lower unemployment duration and recurrence? », IZA discussion paper, $\mathrm{n}^{\circ} 1796$.

Crépon B., Gurgand M., Kamionka T., Lequien L. (2011), Is counseling welfare recipients cost-effective? Lessons from a random experiment, document de travail Crest.

Crépon B., Duflo E., Gurgand M., Rathelot R., Zamora P. (2013), « Do labor market policies have displacement effects? Evidence from a clustered randomized experiment ", The quarterly journal of economics, vol. $128, \mathrm{n}^{\circ} 2$, pp. 531-580.

DARES (2012), «Demandeurs d'emploi inscrits et offres collectées par Pôle Emploi en juin $2012 »$, Dares indicateurs, $\mathrm{n}^{\circ} 2012-50$.

Divay S. (2010), «Nouveaux opérateurs privés du secteur public de l'emploi : les pratiques des conseillers sontelles novatrices?», Travail et emploi, $\mathrm{n}^{\circ}$ 119, pp. 37-49.

FERracci M. (2007), «Améliorer le service public de l'emploi: ce que disent les faits», Revue française d'économie, vol. 21, n 3, pp. 75-135.

Ferracci M., Jolivet G., van den Berg G. J. (2013), « Evidence of treatment spillovers within markets ", à paraître dans la Review of economics and statistics.

Gratadour C., Le Barbanchon T., «Les expérimentations d'accompagnement renforcé de l'Unédic et de l'ANPE : contenu des accompagnements et opinion des bénéficiaires », Premières synthèses, ${ }^{\circ}$ 41.2.

HäGGLund P. (2009), « Experimental evidence from intensified placement efforts among unemployed in Sweden », IFAU Working Paper, n 16.

Hainmueller J., Hofmann B., Krug G., Wolf K. (2011), «Do lower caseloads improve the effectiveness of active labor market policies? New evidence from German employment offices ", MIT political science department research paper, $\mathrm{n}^{\circ}$ 2011-22. 